\title{
Propiedades psicométricas de un instrumento en construcción para evaluar la inteligencia emocional en alumnos de secundaria y la universidad ${ }^{*}$
}

\section{Psychometric properties of a test under construction to assess emotional intelligence in high school and college students}

\author{
Federico Pulido Acosta* \\ ORCID: 0000-0001-7892-6696 \\ Universidad de Granada, España \\ Francisco Herrera Clavero \\ ORCID: 0000-0002-6888-8769 \\ Universidad de Granada, España \\ Recibido: 12 de abril de 2016 \\ Revisado: 1 de junio de 2016 \\ Aceptado: 13 de septiembre de 2016
}

\section{Resumen}

Existe un enorme interés por el desarrollo de programas de educación emocional, programas que deben ser evaluados de manera adecuada. Por eso este trabajo tiene como objetivo elaborar un cuestionario para evaluar la Inteligencia Emocional (IE), siguiendo el modelo que la considera como un conjunto de habilidades, utilizando como referencia el MSCEIT (Mayer Salovey y Caruso, 2009) y destinado a una muestra de alumnos de Secundaria y la universidad. Para esto se contó con 782 participantes, procedentes de 5 centros distintos. El $60.6 \%$ fueron chicas, el $39.4 \%$ varones, el $53.7 \%$ musulmanes, el $46.3 \%$ cristianos. Como instrumentos de evaluación se emplearon un test elaborado para la evaluación de la IE, junto con una adaptación propia de la EHS (Gismero, 2000). Los resultados obtenidos reflejan niveles de fiabilidad aceptables y las dimensiones teóricas esperadas para ambos cuestionarios, apareciendo intercorrelaciones positivas entre los totales y las dimensiones que los conforman. Las correlaciones fueron también positivas y significativas entre ambas escalas. Este cuestionario se muestra como instrumento adecuado para evaluar estas capacidades tras realizar algunas modificaciones en trabajos futuros.

Palabras clave: Inteligencia Emocional, desarrollo afectivo, instrumento de evaluación, educación emocional. 


\section{Abstract}

In the field of education there is high interest in emotional education programs. These programs must be evaluated to check their efficiency. The objective of this work is to develop a questionnaire to assess Emotional Intelligence (El), following the model which conceives it as a set of skills, using the MSCEIT (Mayer et al., 2009) as reference; this questionnaire used a sample of high school and college students. We focused on 782 participants from 5 different centers; $39.4 \%$ are boys and 60.6\% girls; $53.7 \%$ are Muslim and $46.3 \%$ Christian. The techniques used were a test developed to assess El in teenagers and a similar adaptation of EHS (Gismero, 2000). The results show acceptable levels of reliability and also our expectations about theoretical dimensions were confirmed for both questionnaires. There are positive correlations between totals and their constituent dimensions. We found positive and significant correlations between both scales. Therefore, this questionnaire is an appropriate tool to evaluate these capabilities after making some modifications in future works.

Keywords: Emotional Intelligence, assessment tool, emotional education.

\section{Introducción}

La cambiante actualidad obliga a los Sistemas Educativos a emprender retos cada vez más profundos y audaces, para adaptarse a nuevas formas culturales, sociales y políticas de manera vertiginosa, con el fin último de que el alumnado pueda aprender y desarrollar nuevas habilidades y capacidades que le permita afrontar el futuro con relativa esperanza (Codero y Manchón, 2014). Tradicionalmente, se tendía a vivir como si todo dependiera de nuestro exterior, de elementos que están fuera de nosotros mismos, llegando incluso a rechazar la importancia de ese mundo interior, al margen de la Inteligencia Racional. Dentro de esos elementos internos, los estados emocionales comienzan a adquirir una importancia cada vez mayor, van haciéndose un hueco en las más diversas áreas de la vida. Emociones e Inteligencia Racional han sido consideradas como áreas opuestas de la vida mental, sin embargo, en los últimos años se han aproximado entre sí, hasta tal punto que se plantea el manejo emocional como un conjunto de aptitudes y habilidades, de manera idéntica a este segundo elemento.

Cada vez son más las voces que cuestionan la exclusividad de la Inteligencia Racional como factor más influyente para alcanzar el éxito personal, social, académico y profesional, entendiendo que existen otros que también habría que tener en cuenta, como por ejemplo, las inteligencias emocional (Filella, Pérez, Agulló y Oriol, 2014) y social.

Actualmente existe un enorme interés por el desarrollo de programas de educación emocional, con independencia de la etapa educativa a la que este vaya dirigida (Mestre, Guil, Martínez-Cabañas, Larrán y González, 2011). El desarrollo de todos los componentes emocionales, trabajados en este tipo de experiencias, es un elemento favorecedor para el desarrollo en la etapa infantojuvenil, contribuyendo a la mejora de pautas de pensamiento y comportamientos acordes a las normas sociales, al mismo tiempo que un importante preventor de las conductas violentas dentro del ámbito educativo (Gorostiaga y Balluerka, 2014), algo que redunda en el propio beneficio durante la etapa adulta. Las competencias emocionales son además importantes en el proceso de socialización de los más jóvenes, mejorando su rendimiento académico. Todo esto se podrá cumplir si los programas resultan adecuados para las diferentes etapas, por eso los elementos desarrollados deben ser evaluados de manera correcta, lo que implica el uso de instrumentos adecuados (López-Gómez, Hervás y Vázquez, 2015).

Como concepto integrador de todas las capacidades socioemocionales aparece la Inteligencia Emocional, entendida como la habilidad para ma- 
nejar el propio repertorio emocional, pasando el éxito del ámbito cognitivo al ámbito emocional. Resulta evidente que la manera de entenderla se centra en esta en cuanto a un conjunto de habilidades (no de forma percibida) que permiten razonar sobre las emociones y emplearlas para la mejora de los procesos cognitivos. A pesar de esto, las diferencias son claras entre Inteligencia Emocional e Inteligencia Racional, en línea con en el estudio realizado por Pérez y Castejón (2006), donde se indica la independencia de ambas y se desligan claramente. De esta manera, la Inteligencia Emocional se relaciona con las aptitudes con las que las personas ejecutan las tareas y resuelven sus problemas emocionales, en lugar de limitarse a una valoración subjetiva y de manera autopercibida. Son aptitudes reales para resolver problemas emocionales (Mayer et al., 2009). A la vez, esta concepción permite que se pueda hablar de un conjunto de habilidades específicas que integran la IE (Pulido y Herrera, 2015), englobando las habilidades de conocimiento de sí mismos (entendido como la adecuada identificación y comprensión de las emociones propias), autocontrol (manejo de las propias emociones) y motivación (relacionada con el impulso director para el desarrollo de una tarea y cómo una emoción puede facilitar el pensamiento y la ejecución). Estas tres capacidades integran la inteligencia intrapersonal, mientras que la empatía (capacidad de identificar y comprender las emociones en los demás) y Habilidades Sociales (habilidades que permiten la adecuada interacción con los demás de acuerdo con la situación emocional).

Teniendo en cuenta lo comentado, se pretende encontrar un instrumento válido para llevar a cabo la evaluación de las mencionadas capacidades, con intención de hacer más adecuados los programas de educación emocional. Se pueden distinguir dos tipos de instrumentos para evaluar la IE: el primero se refiere a cómo las emociones y el pensamiento interactúan, como el de Mayer et al. (2009). Este tipo de cuestionarios se acercan a la concepción de Inteligencia Emocional antes comentada, sin embargo, se centran en la evaluación de este conjunto de aptitudes exclusivamente en una población adulta, existiendo un vacío en la medida de las mismas en poblaciones juveniles, las cuales constituyen un importante blanco de este tipo de programas educativos centrados en las emociones. Los modelos percibidos (autopercepción de la IE), utilizan inventarios de autoinforme basándose en la popular concepción de la IE de Goleman (1996).

Otro de los aspectos más relevantes en relación con la IE es la gran controversia existente con respecto a la influencia del género en esta. Desde este punto de vista, se parte de diferentes trabajos que plantean que las mujeres manifiestan niveles superiores (Billings, Downey, Lomas, Lloyd y Stough, 2014; Pulido y Herrera, 2015), lo que no hace otra cosa que alimentar el estereotipo de que las mujeres son más "emocionales" que los varones. Sin embargo, en otros estudios se demuestra que las diferencias en función del género están mediatizadas por factores diferentes. Entre estos factores se sugiere la variable edad (Fernández-Berrocal, Cabello, Castillo y Extremera, 2012) o la identidad de género (Gartzia, Aritzeta, Balluerka y Barberá, 2012).

Considerando todo lo mencionado, se presenta el estudio llevado a cabo con una muestra de adolescentes y adultos universitarios, en la ciudad de Ceuta, donde conviven principalmente personas de cultura cristiana y musulmana. Como característica específica, la población árabe musulmana, presenta una procedencia marroquí con un altísimo nivel de analfabetismo y una elevada natalidad, así como un estatus socioeconómico y cultural bajo, serios problemas de enculturación y bilingüismo (integración por la comunicación) y con una presencia muy marcada de su religión, el islam, en sus vidas (Herrera, 2000). Esto nos lleva a establecer una clara relación entre la cultura y la religión.

Los objetivos del trabajo han sido elaborar y validar un instrumento para evaluar la Inteligencia Emocional, considerada como un conjunto de habilidades para una población juvenil (no contemplada en el cuestionario de referencia) y universitaria, así como conocer y analizar las relaciones de la Inteligencia Emocional con las Habilidades Sociales, determinando si se pueden incluir estas últimas dentro de la Inteligencia Emocional. 


\section{Método}

\section{Participantes}

Para llevar a cabo esta investigación se seleccionó una muestra integrada por 782 participantes repartidos entre 5 centros educativos, que reflejan las características de nuestro contexto pluricultural. Para preservar la identidad del alumnado, se emplearon nombres en clave para hacer referencia a cada uno de los IES. Considerando la etapa educativa, la muestra se dividió entre un $28.8 \%$ de alumnado universitario y un $71.2 \%$ correspondiente a secundaria. Las edades de la muestra estaban comprendidas entre los 12 y los 47 años $(M=17.18$, D.T. $=4.67$, Rango=12-47). Describiendo la muestra, en función de la variable género, apa- rece un mayor porcentaje de mujeres, siendo el $39.4 \%$ hombres y el resto $(60.6 \%)$ mujeres. Considerando la cultura (que se corresponde con la religión), el grupo mayoritario es el de participantes pertenecientes a la cultura-religión musulmana. Estos constituyen el $53.7 \%$ de la muestra. El $46.3 \%$ eran cristianos. Atendiendo al estatus, el $4.5 \%$ de la muestra identificó su nivel como bajo. Fueron menos los que se identificaron como pertenecientes a un nivel alto (3.5\%). El $37 \%$ corresponde al estatus medio-bajo y el $55 \%$ al medio. Los participantes se seleccionaron por el método de muestreo no probabilístico por conveniencia, incidental o casual. Se seleccionó, aleatoriamente, un curso (desde primero de ESO a segundo de bachillerato) por cada uno de los centros. Lo mismo ocurrió con el alumnado universitario. El error muestral fue del $3 \%$.

Tabla 1.

Muestra en función de variables sociodemográficas.

\begin{tabular}{llccc}
\hline \multirow{2}{*}{ Variables sociodemográficas } & \multicolumn{3}{c}{ Media descriptiva } \\
\cline { 3 - 5 } & & Media & D.T. & Rango \\
\cline { 3 - 5 } Edad & & 17.18 & 4.67 & $12-47$ \\
\hline \multirow{2}{*}{ Etapa educativa } & Secundaria & 557 & $\%$ \\
\cline { 2 - 5 } & Universidad & 225 & 28.8 \\
\hline \multirow{2}{*}{ Sexo } & Hombres & 308 & 39.4 \\
\cline { 2 - 5 } & Mujeres & 474 & 60.6 \\
\hline \multirow{2}{*}{ Cultura/religión } & Cristianos & 362 & 46.3 \\
\cline { 2 - 5 } & Musulmanes & 420 & 53.7 \\
\hline \multirow{3}{*}{ Estatus } & Bajo & 35 & 4.5 \\
\cline { 2 - 5 } & Medio-bajo & 289 & 37 \\
\cline { 2 - 5 } & Medio & 431 & 55 \\
\cline { 2 - 5 } & Alto & 27 & 3.5 \\
\hline
\end{tabular}

Fuente: elaboración propia. 


\section{Instrumento}

La intención fue la de crear un instrumento que permitiera evaluar la Inteligencia Emocional como conjunto de habilidades específicas. Por tanto, este cuestionario pide al sujeto que resuelva problemas emocionales organizados en diferentes secciones. Para ello se tomó como referencia y punto de partida el MSCEIT (Test de Inteligencia Emocional de Mayer et al., 2009), adaptándolo a la población juvenil. El instrumento de referencia da una visión integradora de la Inteligencia Emocional, presentándola como un grupo de aptitudes y no de manera autopercibida. El problema es que no es apto para su uso en la población juvenil. Al mismo tiempo, se pretende incluir entre las capacidades evaluadas (de acuerdo con la concepción de IE defendida en el apartado teórico) las competencias sociales, no consideradas en el cuestionario de referencia. El instrumento elaborado pretende medir un área o capacidad unitaria (IE), que a su vez se divide en diferentes capacidades específicas (motivación, empatía, autocontrol, autoconcepto). Para llevar a cabo la elaboración del instrumento se contó con la opinión de dos expertos en los campos de la psicología, las emociones, la educación, la evaluación y la estadística. Partiendo del modelo presente en el MSCEIT (dividido en 2 áreas, 4 ramas y 8 tareas) se siguió la misma estructura del cuestionario original, respetando el número de secciones del mismo (exceptuando la $G$, que se suprimió por su excesiva dificultad para el alumnado juvenil). En ellas aparecen apartados para la identificación de emociones en gestos faciales y en diferentes fotografías (secciones A y E del cuestionario original), la comprobación de la utilidad de diferentes emociones en situaciones determinadas (sección B), la selección de la emoción que más se ajuste a cada caso (sección C), la respuesta a cuestiones emocionales acordes a determinadas historias (sección $\mathrm{D}$ y $\mathrm{H}$ ) y la relación de diferentes emociones con acciones concretas (sección F); ajustando las diferentes secciones a la del nivel alumnado. Estas corresponden a cada una de las 8 diferentes tareas que aparecen en el cuestionario original. En este instrumento, la sección eliminada $(G)$ fue sustituida por un apartado para evaluar las competencias sociales del sujeto, aprovechando la idea de incluir ítems que evaluasen estas capacidades, no considerados en el cuestionario original.
Con respecto a las diferentes dimensiones, aparecen factores relacionados con la capacidad del sujeto para percibir emociones con precisión (rama 1: percepción emocional en el cuestionario original), al que se vinculó con el conocimiento de sí mismos. Otra de las dimensiones del cuestionario original se refiere a la capacidad de las emociones para facilitar el pensamiento, utilizándolas en procesos cognitivos (facilitación emocional). En nuestro cuestionario se agruparon por el factor motivación. La rama 3 del cuestionario original (comprensión emocional) quedó dividida, en este caso, en dos factores. Así para la comprensión de información emocional se diferenció entre las propias (autoconcepto) y las ajenas (empatía). La 4 y última rama del cuestionario de referencia se relaciona con la capacidad para manejar las propias emociones, modulando los sentimientos en uno mismo (manejo emocional). En nuestro caso se empleó el término autocontrol. El número total de ítems en el cuestionario fue de 59 , distribuido entre los diferentes factores. Para el sistema de respuestas por parte de los participantes, depende de la sección a la que se preste atención. De esta manera, la respuesta es a través de una escala tipo Likert (secciones $\mathrm{A}, \mathrm{C}, \mathrm{F}$ y $\mathrm{G}$ ) de 4 puntos (con la intención de eliminar la tendencia a contestar la respuesta central se usó un número par de posibilidades) o bien a través de la selección de la emoción que más se adapta a cada situación, imagen o persona (B, D y E) de un listado de 4 diferentes.

Para las Habilidades Sociales se empleó una adaptación de la EHS (Escala de Habilidades Sociales, Gismero, 2000). Este instrumento quedó integrado por 32 ítems (se suprimió 1 de la versión original), reagrupados en 6 factores. Para contestar se utilizó una escala tipo Likert de 4 alternativas. Los factores se relacionan con la asertividad, la expresión de opiniones, la interacción con personas del género opuesto, la manifestación de disconformidad, la expresión de enfado y la realización de peticiones.

\section{Procedimiento}

Tras solicitar y obtener las pertinentes autorizaciones en la Dirección Provincial de Educación (también en el decanato de la facultad), se ha- 
bló con el equipo directivo de los centros que voluntariamente participaron en esta experiencia. Una vez hecho este paso inicial, se redactaron los documentos para recoger por escrito el consentimiento de los padres de los menores que constituirían la muestra (la mayoría de los participantes). En cada uno de los diferentes centros se pasaron los cuestionarios. Se respetó escrupulosamente la confidencialidad, contestando al cuestionario los alumnos que quisieron hacerlo de manera voluntaria. Todos los cuestionarios fueron pasados por el investigador, una persona con dominio en el ámbito de la psicología, la educación y la psicometría, en presencia del profesor de cada aula. Antes de contestar a los cuestionarios, se explicó el modo en el que debían contestar al mismo, haciendo un ejemplo inicial para asegurar el entendimiento por parte de la muestra (lógicamente no contabilizado en el cuestionario). Después de esto, los alumnos contestaron por sí mismos. Se intentó reducir al máximo la influencia de elementos externos que pudieran dificultar la concentración del alumnado, dentro de cada una de las clases correspondientes, configurando un entorno tranquilo y libre de distracciones en función de sus posibilidades. Finalmente, antes de la entrega, se aseguró que ninguno de los participantes dejara ítems sin contestar. La duración de la prueba fue de unos 45 minutos (incluyendo las cuestiones relativas a las HHSS).

Una vez obtenidos los datos y construida la base, se llevó a cabo el pertinente análisis estadístico. Se comenzó por el análisis de la consistencia interna del cuestionario. Para evaluar la fiabilidad se emplearon la prueba a de Cronbach junto con la prueba de dos mitades de Spearman-Brown. La fiabilidad se comprobó tanto para el cuestionario, como para cada uno de los factores (5) obtenidos. Posteriormente se aplicaron cálculos para comprobar la validez factorial, empleando un Análisis Factorial Confirmatorio (AFC), por medio del análisis de varianza de componentes principales con rotación varimax. Para asegurar la validez del modelo jerárquico propuesto, se aplicaron cálculos correlacionales entre el cuestionario (IE) y cada uno de los factores del mismo. De igual manera, se emplearon estos cálculos para comprobar la relación entre la IE y las Habilidades Sociales, intentando justificar la pertinencia de incluirlas como integrantes de la primera (algo hecho desde el punto de vista teórico). Para finalizar, se comprobó la existencia de diferencias estadísticamente significativas empleando la prueba ANOVA, con pruebas post hoc, considerando las variables sociodemográficas como independientes, así como las puntuaciones totales y factoriales tanto de la Inteligencia Emocional como de las Habilidades Sociales.

\section{Resultados}

Se comienza con los resultados que hacen referencia a la consistencia interna del instrumento. La fiabilidad de la medida de la Inteligencia Emocional, a través del cuestionario elaborado, en su versión inicial, se evaluó, en primer caso a través de la prueba a de Cronbach. Así, la consistencia interna del cuestionario (IE) fue de .830. Para el primer factor: motivación, su valor fue de .829 . La del segundo factor: empatía, fue de .714. El tercer factor, que hace referencia al autocontrol obtuvo una fiabilidad de .688. Para el factor autoconcepto fue de .653 y para el factor final: conocimiento de sí mismos, fue el más bajo, ya que se obtuvo solo .642. En un segundo análisis se procedió a realizar la prueba de dos mitades de Spearman-Brown. Este coeficiente fue de .814 para el cuestionario, incluyendo todos los ítems. Para los factores fue de .752, .663, .682, .574 y .621 respectivamente (siguiendo el orden en el que se han comentado anteriormente). Por otro lado, para las cuestiones relativas a las Habilidades Sociales, la consistencia interna viene especificada por un a de Cronbach de .802 y un .773 en el coeficiente de Spearman-Brown. Los valores de la primera prueba (a de Cronbach) quedan recogidos en la tabla 2. 
Tabla 2.

Valores de la prueba a de Cronbach en cuestionario y factores.

\begin{tabular}{|c|c|c|}
\hline \multicolumn{3}{|c|}{ CONSISTENCIA INTERNA DEL CUESTIONARIO } \\
\hline Inteligencia Emocional & a de Cronbach & .830 \\
\hline \multicolumn{3}{|c|}{ CONSISTENCIA INTERNA DE LOS FACTORES } \\
\hline Motivación & a de Cronbach & .829 \\
\hline Empatía & a de Cronbach & .714 \\
\hline Autocontrol & a de Cronbach & .688 \\
\hline Autoconcepto & a de Cronbach & .653 \\
\hline Conocimiento de sí mismos & a de Cronbach & .642 \\
\hline \multicolumn{3}{|c|}{ CONSISTENCIA INTERNA HABILIDADES SOCIALES } \\
\hline Habilidades Sociales & a de Cronbach & .802 \\
\hline
\end{tabular}

Fuente: elaboración propia.

En el apartado relacionado con la varianza factorial se emplearon diferentes Análisis Factoriales Confirmatorios (AFC). En este sentido, los factores obtenidos fueron 5 . El primero de los 5 factores, relacionado con la motivación incluye 20 ítems, que representan el $13.582 \%$ de la varianza explicada. En los ítems que forman parte de este factor aparecen cuestiones del tipo: "¿Qué estado de ánimo es mejor cuando estamos preparando una fiesta de cumpleaños?, o ¿Qué estado de ánimo es útil cuando estamos haciendo un examen?". A continuación el participante responde a través de una casilla en una escala, tipo Likert, en relación con emociones concretas (enfado, miedo, alegría). El segundo de los factores está conformado por 14 ítems (representan el 11.162 \% de la varianza explicada). Este hace referencia a la capacidad del sujeto para la identificación de emociones en otras personas (empatía), dentro de la que aparecen ítems que piden al sujeto que se fije en una serie de imágenes y a continuación se le indica que marque una de una lista de emociones vinculadas a cada una de esas imágenes (por ejemplo miedo, alegría, vergüenza). El tercer factor es el autocontrol emocional o capacidad del sujeto para expresar sus emociones en su justa medida y controlarlas de manera precisa. Son 13 los ítems que lo conforman y representan el 4.447 $\%$ de la varianza explicada. "José estaba muy triste. Había perdido su equipo de fútbol. ¿Cómo ayudarían las siguientes situaciones a que se sintiera mejor?" A continuación se le indican un conjun- to de emociones para que elija, con una escala Likert cuánto de cada una de ellas. Esta es una de las cuestiones que el alumno debe responder dentro de este factor. El cuarto factor, vinculado a la identificación de emociones en uno mismo (autoconcepto) representa el 2.785 \% de la varianza explicada. Integran 7 ítems en los que las cuestiones más importantes, dentro de este factor, piden al sujeto que indique qué emoción sentiría si fuera el protagonista de cada una de las historias que se le plantean (por ejemplo: "fuiste al cine, a ver una película de miedo. Después de verla, estabas muy asustado y no podías dormir. ¿Cómo ayudarían las siguientes situaciones a que te tranquilizaras y pudieras dormir?"). A continuación se le dan a escoger una serie de situaciones (usar técnicas de relajación, pensar en cosas diferentes, ver películas similares). Finalmente, el quinto y último factor, hace referencia al conocimiento de uno mismo, constituido por 5 ítems, que representan el 2.677 \% de la varianza explicada. Dentro de este factor destacan los ítems en los que se indica al sujeto que imagine una serie de situaciones (del tipo: "un compañero te gasta una broma y el profesor te echa de clase a ti", o "estás en el bosque y ves acercarse lentamente un oso") y a continuación se le indica cuánto de cada emoción indicada (miedo, alegría, sorpresa, vergüenza) sentiría en ese momento. Entre todos los factores suman una varianza explicada total del $34.653 \%$. Con respecto a las cuestiones relacionadas con las Habilidades Sociales, se encontraron seis catego- 
rías (factores). El primero de estos seis factores (el factor 1 con una varianza explicada de 15.451 \%) está integrado por 10 ítems y relacionado con la asertividad. El segundo factor (el factor 2 refleja una varianza explicada del $5.221 \%$ ) queda conformado por 8 ítems, relacionados con la expresión de opiniones. El factor 3, que integra 4 cuestiones relativas a la interacción con personas del género opuesto, representa el 4.986 \% de la varianza explicada. El factor 4, relativo a la disconformidad en diferentes circunstancias, está constituido por 5 ítems, que refieren el $4.665 \%$ de la varianza explicada. El factor 5 (varianza explicada de 3.852 $\%)$ está conformado por 2 cuestiones relativas a la de expresión de enfado. El último factor (6) se relaciona con la realización de peticiones, con 3 ítems y dando una varianza explicada de $3.62 \%$. Esto da una varianza explicada total del $37.795 \%$. Todos los resultados en este apartado aparecen resumidos en la tabla 3.

Tabla 3.

Varianza e ítems de la agrupación por factores de los cuestionarios utilizados.

\begin{tabular}{|c|c|c|c|c|}
\hline \multicolumn{5}{|c|}{ VARIANZA DE FACTORES DE CUESTIONARIO IE } \\
\hline & FACTOR & N. ${ }^{\circ}$ ítems & V. explicada & Suma total \\
\hline \multirow{5}{*}{ 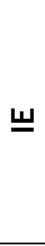 } & Motivación & 20 & $13.582 \%$ & \multirow{5}{*}{$\begin{array}{l}59 \text { ítems } \\
34.653 \% \text { en la varian- } \\
\text { za total explicada }\end{array}$} \\
\hline & Empatía & 14 & $11.162 \%$ & \\
\hline & Autocontrol & 13 & $4.447 \%$ & \\
\hline & Autoconcepto & 7 & $2.785 \%$ & \\
\hline & Conocimiento de sí mismos & 5 & $2.677 \%$ & \\
\hline \multicolumn{5}{|c|}{ VARIANZA DE FACTORES DE CUESTIONARIO HHSS } \\
\hline \multirow{6}{*}{$\begin{array}{l}\text { 呈 } \\
\text { 至 }\end{array}$} & Asertividad & 10 & $15.451 \%$ & \multirow{5}{*}{$\begin{array}{l}32 \text { ítems } \\
37.795 \% \text { en la varian- } \\
\text { za total explicada }\end{array}$} \\
\hline & Opiniones & 8 & $5.221 \%$ & \\
\hline & Interacción género opuesto & 4 & $4.986 \%$ & \\
\hline & Disconformidad & 5 & $4.665 \%$ & \\
\hline & Peticiones & 2 & $3.852 \%$ & \\
\hline & Enfado & 3 & $3.62 \%$ & \\
\hline
\end{tabular}

Fuente: elaboración propia.

De la misma manera que hace el MSCEIT, el cuestionario elaborado pretende medir un área unitaria, la Inteligencia Emocional, que a su vez se subdivide en una serie de habilidades específicas. Para que el planteamiento jerárquico propuesto sea válido, es necesario que las diferentes puntuaciones correlacionen positivamente entre sí. La correlación fue significativa al nivel $p=.01$, tal y como era de esperar. Las correlaciones más bajas (aunque cumpliendo lo comentado) se dan entre la Inteligencia Emocional y el factor empatía (.495). Fuera de ellas, los resultados se mueven entre el máximo (.703), encontrado en el factor motiva- ción y el mínimo (.617) obtenido entre la Inteligencia Emocional y el conocimiento de sí mismo. Por otro lado, los mismos resultados comentados para la Inteligencia Emocional y todos los factores se repiten considerando las intercorrelaciones de los factores entre sí. En todos los casos el nivel de significación es de al menos $p=.01$, siendo en todos los casos positivos. En este sentido, los niveles son más bajos que los reflejado para los totales (algo esperado), moviéndose entre los más altos (.427) encontrados en las correlaciones entre autocontrol y empatía y los más bajos (.090) encontrados entre la empatía y la motivación. 
Tabla 4.

Correlaciones factores IE.

\begin{tabular}{|c|c|c|c|c|c|c|}
\hline & IE & Motiv. & Empa & Aucont & Aucepto & Conoci. \\
\hline Inteligencia Emocional & 1 & & & & & \\
\hline Motivación & .703 & 1 & & & & \\
\hline Empatía & .495 & .090 & 1 & & & \\
\hline Autocontrol & .691 & .197 & .427 & 1 & & \\
\hline Autoconcepto & .621 & .422 & .175 & .294 & 1 & \\
\hline Conocimiento de sí mismos & .617 & .291 & .290 & .397 & .355 & 1 \\
\hline
\end{tabular}

Fuente: elaboración propia.

Exactamente lo mismo que lo comentado para la Inteligencia Emocional, se ha realizado con la adaptación realizada de la EHS. En este caso, también aparece un área unitaria (Habilidades Sociales) dividida en un conjunto de capacidades más concretas. En este caso, las correlaciones encontradas entre las Habilidades Sociales totales y los factores que la conformaban fueron de nuevo significativas al nivel $p=.01$, tal y como era de esperar. En todos los casos, desde aquellos en los que se obtiene una puntuación más alta $(.813$ se encontró entre las Habilidades Sociales y la asertividad), hasta aquellos en los que los resultados son más bajos (.237 fue el resultado de la correlación entre las Habilidades Sociales y la realización de peticiones) se cumple lo comentado. Por su parte, resultados similares se obtienen entre los diferentes factores que conforman las Habilidades Sociales. En esta ocasión, en la mayoría de los casos, las interacciones vuelven a cumplir el nivel de $p=.01$. Se mueven entre los niveles más bajos (.120) encontrados en las correlaciones entre la asertividad y la realización de peticiones, y los más altos (.478) encontrados entre la asertividad y la manifestación de opiniones. Todo ello se cumple en todos los factores, exceptuando el último (peticiones), en el que aparecen correlaciones no significativas, encontradas entre este factor y la asertividad (.009), la interacción con el género opuesto $(.024)$ y manifestación de enfado $(-.058)$.

Finalmente se comentan las correlaciones existentes entre las puntuaciones de Inteligencia Emocional y las Habilidades Sociales, así como cada uno de sus factores. En esta ocasión las diferencias son significativas al nivel $p=.01$ para las correlaciones entre la Inteligencia Emocional y los factores manifestación de enfado (.097) y asertividad (.092), y al nivel $p=.05$ entre la Inteligencia Emocional y las Habilidades Sociales totales (.060), la disconformidad $(.060)$ y la realización de peticiones. Sin embargo, mientras que para las dos primeras son positivas, para la última es negativa ( -.063 , lo que indica la ausencia de relación). El resto de correlaciones no fueron estadísticamente significativas. A pesar de ello, la correlación entre la Inteligencia Emocional y las Habilidades Sociales totales indica relación entre escalas. Todos los resultados comentados aparecen resumidos en la tabla 5. 
Tabla 5.

Correlaciones factores HHSS.

\begin{tabular}{|c|c|c|c|c|c|c|c|}
\hline & IE & HHSS & Aserti. & Opini. & I.G.O. & Disc. enfad & Peticio \\
\hline Inteligencia Emocional & 1 & & & & & & \\
\hline Habilidades Sociales & $.060^{* *}$ & 1 & & & & & \\
\hline Asertividad & $.092^{*}$ & $.813^{*}$ & 1 & & & & \\
\hline Opiniones & .007 & $.798^{*}$ & $.478^{*}$ & 1 & & & \\
\hline Interacción género opuesto & .026 & $.632^{*}$ & $.438^{*}$ & $.359^{*}$ & 1 & & \\
\hline Disconformidad & $.060^{* *}$ & $.627^{*}$ & $.416^{*}$ & $.410^{*}$ & $.281^{*}$ & 1 & \\
\hline Enfado & $.097^{*}$ & $.457^{*}$ & $.302^{*}$ & $.298^{*}$ & $.224^{*}$ & $.246^{*}$ & 1 \\
\hline Peticiones & $-.063^{* *}$ & $.237^{*}$ & $.009 *$ & $.120^{*}$ & $.024^{*}$ & $.121^{*}$ & -.058 \\
\hline
\end{tabular}

La correlación es significante al nivel $0.01^{*}$. La correlación es significativa al nivel $0.05^{* *}$.

Fuente: elaboración propia.

Para finalizar el apartado de resultados, se comentan los de la prueba ANOVA, con la intención de comprobar la existencia de diferencias significativas en función de las variables sociodemográficas. En este sentido, se comentan diferencias significativas considerando la etapa educativa del alumnado como variable factor. De esta mane$\mathrm{ra}$, las diferencias fueron significativas tanto en la IE total $(p=.013)$, motivación $(p=.000)$, empatía $(p=.000)$, autocontrol $(p=.000)$ y conocimiento de sí mismos $(p=.028)$. En todos los casos, la Inteligencia Emocional y sus factores aumentan con la edad, siendo superiores entre los alumnos universitarios (la media es de 117.44 mientras que la de secundaria fue de 104.33). El autoconcepto $(p=.801)$ es el único caso en el que las diferencias no fueron significativas. Lo mismo ocurrió en el caso de las Habilidades Sociales $(p=.000)$, la asertividad $(p=.000)$, la interacción con el género opuesto $(p=.000)$, la disconformidad $(p=.000)$, la expresión de enfado $(p=.000)$ y la realización de peticiones $(p=.028)$. En todos los casos, los alumnos universitarios alcanzan puntuaciones más altas. En la manifestación de opiniones $(p=.902)$, las diferencias no fueron significativas.

Considerando el género, tal y como demuestran los resultados de la prueba ANOVA, se observan diferencias significativas en la Inteligencia Emocional total $(p=.013)$ y en el factor empatía $(p=.000)$, siendo las mujeres (media de 104.98) las que evidencian resultados superiores (hombres 101.37). Para el resto de factores desde la motivación $(p=.808)$, el autocontrol $(p=.346)$, el autoconcepto $(p=.621)$ hasta el conocimiento de sí mismos $(p=.069)$, no se obtuvieron diferencias significativas. En todos los casos en los que se encontraron diferencias significativas son las mujeres las que obtienen resultados superiores. En Habilidades Sociales totales $(p=.000)$, asertividad $(p=.007)$, manifestación de opiniones $(p=.000)$, interacción con el género opuesto $(p=.010)$, expresión de enfado $(p=.000)$ y realización de peticiones $(p=.000)$ las diferencias son significativas, siendo los hombres los que obtienen resultados superiores. Para la disconformidad $(p=.061)$ no fueron significativas.

Teniendo en cuenta el grupo cultural, se reflejan diferencias estadísticamente significativas en los elementos relacionados con la Inteligencia Emocional. Así, en los totales $(p=.000)$, la empatía $(p=.000)$, el autocontrol $(p=.000)$, el autoconcepto $(p=.012)$ y el conocimiento de sí mismos $(p=.000)$, siendo los cristianos los que registran resultados más altos (su media total es de 108.37, la de los musulmanes de 99.41). Las diferencias no fueron significativas en el caso de la motivación $(p=.112)$. Las diferencias son también significativas para las Habilidades Sociales $(p=.018)$, la manifestación de opiniones $(p=.031)$ y la expresión de enfado 
$(p=.000)$. No fueron significativas, sin embargo, para la asertividad $(p=.089)$, la interacción con personas del género opuesto $(p=.144)$, la disconformidad $(p=.635)$ y la realización de peticiones $(p=.658)$. Vuelven a ser los musulmanes los que reflejan resultados más bajos.

Las diferencias fueron significativas en los niveles de Inteligencia Emocional total $(p=.000)$, la motivación $(p=.000)$, la empatía $(p=.006)$, el autocontrol $(p=.000)$, el autoconcepto $(p=.000)$ y el conocimiento de sí mismos $(p=.000)$. El nivel alto se asocia con mayores habilidades emocionales (el alto es en el que aparecen resultados más elevados -113.95-, seguido del medio -108.27-, siendo prácticamente idénticas y más bajas las medias del estatus medio-bajo y del bajo). No fueron significativas para las Habilidades Sociales totales $(p=.498)$, la asertividad $(p=.150)$, la manifestación de opiniones $(p=.816)$, la interacción con personas del género opuesto $(p=.623)$, la disconformidad $(p=.949)$, la expresión de enfado $(p=.548)$ y la realización de peticiones $(\mathrm{p}=.281)$.

\section{Conclusiones}

Partimos en el presente estudio con la idea de hacer una primera validación inicial de un instrumento para evaluar la Inteligencia Emocional, con la idea de medir esta habilidad en una población juvenil y universitaria, basándonos en las características del MSCEIT (Mayer et al., 2009). Los niveles de consistencia interna del instrumento fueron altos para la Inteligencia Emocional ( $\alpha=.830$; coeficiente de Spearman-Brown=.814) y aceptables para cada uno de los factores (a desde .829 hasta .642 , coeficiente de Spearman-Brown desde .752 hasta .621). Respecto a la dimensionalidad del instrumento, se obtuvieron 5 factores (motivación, empatía, autocontrol, autoconcepto y conocimiento de sí mismos) que consideran capacidades similares a las del cuestionario de referencia. Entre todos los factores suman una varianza explicada total del 34.653 \% quedando el cuestionario integrado por un total de 59 ítems. Estos resultados, unidos a los referentes a las correlaciones (significativos al nivel $p=.01$ en todos los casos) que se dan entre la Inteligencia Emocional y cada uno de los factores, de la misma manera que los obtenidos para las intercorrelaciones entre factores, apoyan el concepto jerárquico propuesto que parte de la existencia de un área unitaria (Inteligencia Emocional) y una dimensionalidad múltiple (habilidades específicas). La validez convergente del instrumento esperaba obtener y obtuvo correlaciones positivas y estadísticamente significativas entre las dimensiones del instrumento. Se encontraron diferencias significativas por etapa educativa (asociadas a la edad del alumnado), tanto en los totales como en cada uno de los factores. Parece claro que las diferencias encontradas son fruto del desarrollo emocional que caracteriza el proceso evolutivo dentro de la población estudiada, algo ya mencionado en Billings et al. (2014); Brouzos, Misailidi y Hadjimattheou (2014); Pulido y Herrera (2015). Se encontraron diferencias significativas en función del género tanto en los niveles totales como en el factor empatía, teniendo las mujeres resultados más altos (Gartzia et al., 2012). Las diferencias con respecto a la cultura fueron significativas en los totales y todas las categorías, actuando todas en una misma dirección: en todos los casos los cristianos obtienen mejores resultados. Diferencias en las habilidades emocionales entre dos grupos culturales también se encontraron en Pulido y Herrera (2015), donde se señalan las dificultades para la integración socioeducativa (Martínez y Alfageme, 2004) y las diferencias de estatus socioeconómico y cultural (Hernández, Rodríguez y Moral, 2011) como importantes justificantes de estas diferencias, junto con la diversidad encontrada en los núcleos familiares, con estilos de crianza distintos y las importantes diferencias en la lengua materna (Roa, 2006; Pulido y Herrera, 2015) entre ambas etnias, contribuyen a la aparición de problemas de integración socioeducativa del alumnado no autóctono. Esto se ve reforzado también por las diferencias encontradas por estatus, variable íntimamente relacionada con la cultura.

En segundo lugar, también se hizo una primera validación de un instrumento para evaluar las Habilidades Sociales, empleando como referencia la EHS (Gismero, 2000). La fiabilidad encontrada fue elevada en los totales $(\alpha=.802$; coeficiente de Spearman-Brown=.773). Las dimensiones 
encontradas fueron seis (asertividad, manifestación de opiniones, interacción con personas de género opuesto, disconformidad, expresión de enfado y realización de peticiones). Esto da una varianza explicada total del $37.795 \%$, quedando la escala integrada por 32 reactivos. Existen correlaciones significativas entre las Habilidades Sociales y las dimensiones que la conformaban, del mismo modo que entre dichas dimensiones (con la excepción de algunos factores que no se relacionan con la realización de peticiones). Se encontraron diferencias significativas por etapa, género (más los hombres) y cultura (en los totales y algunos factores, teniendo los cristianos mayores puntuaciones).

Las correlaciones resultaron positivas y estadísticamente significativas entre los totales de Inteligencia Emocional y las Habilidades Sociales, de la misma forma que para los factores asertividad y manifestación de enfado. Para el resto de factores las correlaciones no fueron significativas o incluso significativas con sentido negativo. A pesar de ello, empleando esta escala para evaluar las Habilidades Sociales, pueden tener cabida dentro del ámbito de la Inteligencia Emocional.

Finalmente indicar que, para ser la primera validación, los resultados iniciales nos permiten ser optimistas con respecto a los elementos a corregir para depurar este instrumento de evaluación de las habilidades emocionales. Del mismo modo, puede ser un importante cuestionario a emplear dentro del ámbito psicológico, social y, sobre todo, educativo en una población adolescente, superando elementos como la complejidad para leer y entender los ítems en el cuestionario de referencia, destinado a una población adulta. No obstante es importante remarcar que el trabajo se encuentra en sus fases iniciales, presentando importantes limitaciones (fiabilidad a aumentar, varianza explicada por los factores a aumentar, eliminación y reformulación de ítems) lo que deja abierta futuras experiencias dentro de este campo.

\section{Referencias}

Billings, C., Downey, L. A., Lomas, J. E., Lloyd, J., \& Stough, C. (2014). Emotional Intelligence and scholastic achievement in preadolescent children. Personality and Individual Differences, 65, 14-18.

Brouzos, A., Misalidi, P., \& Hadjimatheou, A. (2014). Associations between Emotional Intelligence, socio-emotional adjustment, and academic achievement in childhood: The influence of age. Canadian Journal of School Psychology, 29(2), 83-99.

Codero, J. M., y Manchón, C. (2014). Factores explicativos del rendimiento en educación primaria: un análisis a partir de TIMSS 2011. Estudios sobre Educación, 27, 9-35.

Fernández-Berrocal, P., Extremera, N., \& Ramos, N. (2004). Validity and reliability of the spanish modified version of the trait meta-mood scale. Psychological Reports, 94, 751-755.

Filella, G., Pérez, N., Agulló, M. J., y Oriol, X. (2014). Resultados de la aplicación de un programa de educación emocional en educación primaria. Estudios sobre Educación, 26, 125-147.

Gartzia, L., Aritzeta, A., Balluerka, N., y Barberá, E. (2012). Inteligencia emocional y género: más allá de las diferencias sexuales. Anales de psicología, 28(2), 567-575.

Gismero E. (2000). EHS, Escala de Habilidades Sociales. Madrid: TEA Publicaciones de Psicología Aplicada.

Goleman, D. (1996). Inteligencia Emocional. Barcelona: Kairós. 
Gorostiaga, A., y Balluerka, N. (2014). Evaluación de la empatía en el ámbito educativo y su relación con la inteligencia emocional. Revista de Educación, 364, 12-38.

Hernández, E., Rodríguez, F. J., y Moral, M. V. (2011). Adaptación escolar de la etnia gitana: relevancia de las variables psicosociales determinantes. Apuntes de Psicología, 29(1), 87-105.

Herrera, F. (2000). La inmigración extranjera no comunitaria y la convivencia en contextos concretos: el caso de Ceuta. En Instituto de Estudios Ceutíes, Monografía de los cursos de verano de la Universidad de Granada en Ceuta (12. ${ }^{\text {a }}$ ed., pp. 357-359). Ceuta: Instituto de Estudios Ceutíes-Universidad de Granada.

López-Gómez, I., Hervás, G., y Vázquez. C. (2015). Adaptación de las "Escalas de afecto positivo y negativo" (PANAS) en una muestra general española. Behavioral Psychology, 23(3), 45-52.

Martínez, M., y Alfageme, A. (2004). Integración socioeducativa del alumno gitano en la escuela española. Revista Española de Educación Comparada, 10, 299-324.
Mayer, J., Salovey, P., y Caruso, D. (2009). Test de Inteligencia Emocional de Mayer, Salovey y Caruso. Madrid: TEA Ediciones.

Mestre, J. M., Guil, R., Martínez-Cabañas, F., Larrán, C., y González, G. (2011). Validación de una prueba para evaluar la capacidad de percibir, expresar y valorar emociones en niños de la etapa infantil. Revista Electrónica Interuniversitaria de Formación del Profesorado, 14(3), 37-54. Recuperado de http:// www.aufop.com

Pérez, N., y Castejón, J. L. (2006). Relaciones entre la Inteligencia Emocional y el cociente intelectual con el rendimiento académico en estudiantes universitarios. Revista Electrónica de Motivación y Emoción (R.E.M.E.), 9(22), 1-27.

Pulido, F., y Herrera, F. (2015). Miedo e Inteligencia Emocional en el contexto pluricultural de Ceuta. Anuario de Psicología, 45(2), 249-263.

Roa, J. M. (2006). Rendimiento escolar y "situación diglósica" en una muestra de escolares de educación primaria en Ceuta. Revista Electrónica de Investigación Educativa, 8, 1-15. Recuperado de http://redie.uabc.mx/ vol8no1/contenido-roa.html 